\title{
DAMAGE DETECTION OF BEAM BY NATURAL FREQUENCIES: GENERAL THEORY AND PROCEDURE
}

\author{
NGUYen Tien KHIEM \\ Institute of Mechanics, VAST \\ 264, Doi Can, Hanoi, Vietnam, ntkhiem@imech.ac.vn
}

\begin{abstract}
The frequency equation of single damaged beam has been established for arbitrary boundary conditions that is the main tool for analysis as well as identification of damaged beam by using measured natural frequencies. A procedure for damage detection problem presented in this paper consists of three steps. First, the modelling error is reduced by a model updating procedure, in which the material, geometrical parameters and boundary conditions are updated. Then, measurement data are corrected based on the updated model. Finally, the damage parameters are identified using updated model and corrected measurement data. Theoretical investigation is illustrated by an example.
\end{abstract}

Keywords: damage detection, model updating, beam.

\section{INTRODUCTION}

A damage often occurred on members of structures may cause serious faibure of structures. Therefore, damage must be detected as early as possible. However, the damage detection problem even for one-dimensional structure is still difficult and has not been solved completely. The difficulties arose mostly from the modelling error and measurement noise. Reduction of modelling error has been mentioned in some studies, but less attention is paid for correcting of noised and erroneous measurement data. The aim of this paper is to develop a complete procedure for solving the damage detection problem of beam including correction of not only the model but also the measurement data.

There are different points of view criticising the use of modal parameters in damage detection, especially the case of using only the natural frequencies. Nevertheless, in practice the natural frequencies of structures are measured most easily and accurately in comparison with all other parameters such as the mode shapes for example. Using the other parameters, which are more contaminated by measurement noise, is not sure to improve the result of the damage identification. The author of present work agrees with Messina et al. stated in [1] that "a method capable of predicting the extent as well as the location of damage that requires only the change in the natural frequencies would be welcomed".

Since that discussion, the main focus of this paper is the problem of damage detection for one-dimensional structure using its the analytical model and measured only natural frequencies. The damage is treated as a change in sectional stiffness (or flexibility), which is represented by an equivalent (axial or rotational) spring connecting the both sides of the damage position. In 1978 Adams et al. [2] investigated the case of damage that was modelled by an axial spring (axial damage model), but there is absent a calculating stiffness of the spring. Then transverse (rotational) model of damage has been developed and validated by a general theory of damaged beams [3] that makes it be possible to 
determine the stiffness of the equivalent spring as a function of damage depth. Using the transverse model of damage Rizos et al. [4] have constructed the frequency equation for cantilever beam. Narkis [5] has given the equations for simply supported beam in both the cases of transverse and axial models. Boltezar et al. [6] did the same task for free-free beams with a transverse damage. Masoud et al. [7] considered the case of axially loaded fixed-fixed damaged beam. Nandwana et al [8] developed the theory for stepped cantilevers and Tsai and Wang [9] and Hai-Ping Lin [10] - for Timoshenko beams. While all the listed studies were concerned with a single damage, the multiple damaged beams were investigated by Ruotolo and Surace in [11], Khiem and Lien [12]. Furthermore, Liang [13] has shown that there exists a general form of the frequency equation for the both cantilever and simply supported beams. Morassi [14] has constructed a sensitivity equation of frequencies for beams with elastic supports at the beam ends, but he investigated only the case of symmetry of the supports. In the our paper [15] the frequency equation of damaged beam has been established in general form for all of the classical boundary conditions, that is likely the equation given by Liang. In this paper the general frequency equation of damaged beams obtained in [15] will be developed for elastic end supports since, as shown in reference [16], the idealisation of the boundary conditions can lead to significant shift of natural frequencies.

The works done by Adams et al. [2], Liang [13], Morassi [14] are very important in the field of damage location of beam by natural frequencies. They have shown that the ratio of changes in two frequencies due to damage is independent on the damage magnitude but is a function of only the damage position. Based on this fact Adams et al. have suggested a graphic method to locate the damage by seeking an intersection of curves computed from the shifts of frequencies and mode shapes of undamaged beam. This method has been developed further in $[6,8]$. However, the question as influence of the model error (boundary conditions) and measurement noise on accuracy of the damage location has been still unanswered. The model updating problem before the damage detection is firstly investigated by Adams et al. in [4] who proposed to correct the Young's modulus E as a model parameter to be updated. Moreover, the seeking intersection of curves in presence of the inaccurate model and the measurement error will be very difficult, not yet taking into account the effect of computational error. Therefore, another criterion for locating the damage as well as for determining the damage magnitude is required instead of the graphic one. Ruotolo and Surace have presented in [11] very interesting analysis and comparison in using different objective functions. They have shown that the choosing optimisation principles affects strongly on the result of damage identification because one often may find rather the local than global minimum. There is need of a criterion for finding the global minimum of the chosen objective function. It is interesting to mention the paper of Armon et al. [17] where another criterion named as rank ordering one has been suggested and, as claimed the authors, is robust with respect to measurement error, boundary conditions and to many other factors. Nevertheless, in the paper there is a lack of an explicit algorithm to "measure" the rank ordering of frequency fractional changes and it requires to measure not only frequencies of damaged but also of the undamaged structure that is not realistic from the viewpoint of practical application. This limitation leads to the idea of using not frequency changes but the absolute values of measured frequencies of damaged structures.

In this paper, frst, a general characteristic equation of beams with a single trans- 
verse damage for generalised the boundary conditions, including also the elastic supports is established. This equation contains not only the beam constants, the damage parameters, but also the boundary parameters, that may be used for model updating before the damage detection. The obtained equation can be useful in theoretical investigation, for instance, in sensitivity analysis of frequencies to damage, boundary conditions or to the structural constants and of course it will be the main equation used for damage identification. Next, the beam constants and boundary parameters have been corrected in order to update the model. This model updating and measurement data correction procedure would be rather convenient and simple if the natural frequencies of undamaged beam are available. Otherwise, the raw values of damage parameters have been obtained in the model updating stage together with the model parameters and they will be used to correct the measurement data. The last correction is performed by calculating the natural frequencies from updated model of damaged beam. Using the updated model and corrected measurement data, the damage position and magnitude are identified by solving simple optimisation problem. Thus, the damage detection problem is significantly simplified. The proposed herein generalised procedure for damage detection of beam will be illustrated by a numerical example.

\section{VIBRATION MODEL OF A DAMAGED BEAM}

Consider a beam with the material and geometrical constants $E, I, \rho, A, L$. The equation of transverse vibration of the beam as well known is

$$
E I w^{(I V)}(x, t)+\rho A \ddot{w}(x, t)=0 ;-0.5 \leq x \leq=0.5 .
$$

Introducing the parameters $a=L(\rho A / E I)^{1 / 4}$ and $\lambda=a \sqrt{\omega}$, where $\omega$ - natural frequency of the beam, the mode shape of vibration can be determined by the equation

$$
\phi^{(I V)}(x)-\lambda^{4} \phi(x)=0 ; x \in[-0.5 ;+0.5] .
$$

Suppose that the beam is damaged at a position $s$ and the damage is modelled as a rotational spring of stiffness $K$, connecting beam segments in both sides of the damage position. Following T. G. Chondros and A. D. Dimarogonas et al. [3], we have

$$
K=\frac{1}{C} ; C=\frac{6 \pi\left(1-\nu^{2}\right) h}{E I} I_{c}\left(\frac{\alpha}{h}\right),
$$

where $\nu$ - the Poisson ratio and the function $I_{c}(z)$ for single-edge open damage has the form

$$
\begin{aligned}
I_{c}(z)= & 0.6272 z^{2}-1.04533 z^{3}+4.5948 z^{4}-9.973 z^{5}+20.2948 z^{6} \\
& -33.0351 z^{7}+47.1063 z^{8}-40.7556 z^{9}+19.6 z^{10} .
\end{aligned}
$$

If a parameter $\beta=E I / K L$ is introduced for description of relative flexibility of the spring and called here damage magnitude, then $\beta=0(K=\infty)$ will correspond to the undamaged case and $\beta_{\max }=138.5(\mathrm{~h} / \mathrm{L})$, when $\alpha=h$ - to the completely damaged one. Furthermore, at the damage sit it's must be hold the condition

$$
\begin{array}{ll}
\phi(s-0)=\phi(s+0) ; & \phi^{\prime \prime}(s-0)=\phi^{\prime \prime}(s+0) ; \\
\phi^{\prime \prime \prime}(s-0)=\phi^{\prime \prime \prime}(s+0) ; & \phi^{\prime}(s-0)+\beta \phi^{\prime \prime}(s-0)=\phi^{\prime}(s+0) .
\end{array}
$$


Let the so-called shape functions $L_{i}(z), i=1, \ldots, 4$ be defined so that the functions

$$
\begin{array}{ll}
\phi_{1}(x)=C_{1} L_{1}(\xi)+C_{2} L_{2}(\xi) ; & \phi_{2}(x)=C_{3} L_{3}(\zeta)+C_{4} L_{4}(\zeta) ; \\
\xi=\lambda(x+0.5) ; & \zeta=\lambda(x-0.5)
\end{array}
$$

will satisfy the given boundary conditions. The shape functions $L_{i}(z), i=1, \ldots, 4$, defined below in Appendix I, could depend on the boundary parameters denoted by $b=\left\{b_{i}, i=1, \ldots, 4\right\}$, if the more realistic (elastic) supports are considered. The parameters $b$ are introduced for updating the model by measurement data, the $C_{i}, i=1,2,3,4$ are constants to be determined.

Seeking solution of the equation (2.2) in the form

$$
\phi(x)=\left\{\begin{array}{l}
\phi_{1}(x), \quad-0.5 \leq x \prec s \\
\phi_{2}(x), \quad s \prec x \leq 0.5
\end{array},\right.
$$

after substituting this function into (2.3), for existence of the non-trivial (nonzero) constants $\mathrm{C}$ there must be obtained the characteristic equation

$$
F(\lambda, s, \beta, b)=\operatorname{det}[A]=0,
$$

where

$$
\begin{aligned}
& {[A]=\left[\begin{array}{llll}
L_{1}\left(\xi_{s}\right) & L_{2}\left(\xi_{s}\right) & -L_{3}\left(\zeta_{s}\right) & -L_{4}\left(\zeta_{s}\right) \\
L_{1}^{\prime}\left(\xi_{s}\right)+\lambda \beta L_{1}^{\prime \prime}\left(\xi_{s}\right) & L_{2}^{\prime}\left(\xi_{s}\right)+\lambda \beta L_{2}^{\prime \prime}\left(\xi_{s}\right) & -L_{3}^{\prime}\left(\zeta_{s}\right) & -L_{4}^{\prime}\left(\zeta_{s}\right) \\
L_{1}^{\prime \prime}\left(\xi_{s}\right) & L_{2}^{\prime \prime}\left(\xi_{s}\right) & -L_{3}^{\prime \prime}\left(\zeta_{s}\right) & -L_{4}^{\prime \prime}\left(\zeta_{s}\right) \\
L_{1}^{\prime \prime \prime}\left(\xi_{s}\right) & L_{2}^{\prime \prime \prime}\left(\xi_{s}\right) & -L_{3}^{\prime \prime \prime}\left(\zeta_{s}\right) & -L_{4}^{\prime \prime \prime}\left(\zeta_{s}\right)
\end{array}\right]} \\
& \xi_{s}=\lambda(s+0.5) ; \zeta_{s}=\lambda(s-0.5) .
\end{aligned}
$$

By the simple calculation it is not difficult to verified that the function $F$ can be expressed in the form

$$
F(\lambda, s, \beta, b)=\beta F_{1}(\lambda, s, b)+F_{0}(\lambda, b),
$$

where $F_{1}(\lambda, s, b)=\operatorname{det}\left[A_{1}\right] ; F_{0}(\lambda, b)=\operatorname{det}\left[A_{0}\right]$ and matrices $\left[\mathrm{A}_{1}\right],\left[\mathrm{A}_{0}\right]$ are

$$
\begin{aligned}
{\left[A_{1}\right]=} & {\left[\begin{array}{cccc}
L_{1}\left(\xi_{s}\right) & L_{2}\left(\xi_{s}\right) & -L_{3}\left(\zeta_{s}\right) & -L_{4}\left(\zeta_{s}\right) \\
\lambda L_{1}^{\prime \prime}\left(\xi_{s}\right) & \lambda L_{2}^{\prime \prime}\left(\xi_{s}\right) & 0 & 0 \\
0 & 0 & -L_{3}^{\prime \prime}\left(\zeta_{s}\right) & -L_{4}^{\prime \prime}\left(\zeta_{s}\right) \\
L_{1}^{\prime \prime \prime}\left(\xi_{s}\right) & L_{2}^{\prime \prime \prime}\left(\xi_{s}\right) & -L_{3}^{\prime \prime \prime}\left(\zeta_{s}\right) & -L_{4}^{\prime \prime \prime}\left(\zeta_{s}\right)
\end{array}\right] } \\
{\left[A_{0}\right]=} & {\left[\begin{array}{llll}
L_{1}\left(\xi_{s}\right) & L_{2}\left(\xi_{s}\right) & -L_{3}\left(\zeta_{s}\right) & -L_{4}\left(\zeta_{s}\right) \\
L_{1}^{\prime}\left(\xi_{s}\right) & L_{2}^{\prime}\left(\xi_{s}\right) & -L_{3}^{\prime}\left(\zeta_{s}\right) & -L_{4}^{\prime}\left(\zeta_{s}\right) \\
L_{1}^{\prime \prime}\left(\xi_{s}\right) & L_{2}^{\prime \prime}\left(\xi_{s}\right) & -L_{3}^{\prime \prime}\left(\zeta_{s}\right) & -L_{4}^{\prime \prime}\left(\zeta_{s}\right) \\
L_{1}^{\prime \prime \prime}\left(\xi_{s}\right) & L_{2}^{\prime \prime \prime}\left(\xi_{s}\right) & -L_{3}^{\prime \prime \prime}\left(\zeta_{s}\right) & -L_{4}^{\prime \prime \prime}\left(\zeta_{s}\right)
\end{array}\right] }
\end{aligned}
$$

The independence of the function $F_{0}$ on the parameter $s$ may be easy checked by differentiation of the determinant. Thus, the called frequency equation with respect to the frequency parameter $\lambda$ takes the final form

$$
F(\lambda, s, \beta, b)=\beta F_{1}(\lambda, s, b)+F_{0}(\lambda, b)=0 .
$$


A modification of the equation (2.6) has been derived in [14], [15] for all the classical boundary conditions. The given herein equation is the most general because it is derived for generalised boundary conditions including as the classical ones as well as the more realistic elastic supports. For undamaged beam the frequency equation is much simplified as $F_{0}(\lambda, b)=0$. From the equation (2.6) it may be seen that the ratio

$$
h=F_{1}\left(\lambda_{j}, s, b\right) / F_{0}\left(\lambda_{j}, b\right),
$$

will be a constant for all the vibration modes and depended on only the damage sit, the fact mentioned above in introduction.

\section{MODEL UPDATING AND MEASUREMENT DATA CORRECTION}

Letting $\omega^{*}=\left(\omega_{1}^{*}, \ldots, \omega_{m}^{*}\right)^{T}$ be the $m$ measured frequencies of the beam, we introduce a vector $\Omega=\left(\Omega_{1}, \ldots, \Omega_{m}\right)^{T}=\left(\sqrt{\omega_{1}^{*}}, \ldots, \sqrt{\omega_{m}^{*}}\right)^{T}$, which represent the measured data without any change of their nature themselves. The main parameter to be corrected is $a=L(\rho A / E I)^{\frac{1}{4}}$ and boundary parameters $b=\left(b_{1}, \ldots, b_{n}\right)^{T}$ beside the damage parameter $(s, \beta)$.

In the model of damaged beam described above, the prediction of natural frequencies is obtained by solving the frequency equation with respect to the so-called frequency parameters $\lambda_{j}, j=1, \ldots, m$. The later are functions of the boundary and damage parameters $\lambda_{j}=\lambda_{j}(b, s, \beta)$. On the other hand, for the beam the relationship $\lambda_{j}=a \Omega_{j}$ must be hold for every $j$. So, the predicted and measured frequency parameters must satisfy the equalities

$$
\frac{\lambda_{j}}{\Omega_{j}}=a=\text { const } \quad \forall j=1, \ldots, m,
$$

which are equivalent to

$$
\sum_{j=1}^{m}\left(\lambda_{j}-a \Omega_{j}\right)^{2}=|\lambda|^{2}-2 a \lambda^{T} \Omega+a^{2}|\Omega|^{2}=0,
$$

with the notations $\lambda^{T} \Omega=\left(\sum_{j=1}^{m} \lambda_{j} \Omega_{j}\right) ;|\lambda|^{2} \equiv \lambda^{T} \lambda=\sum_{j=1}^{m} \lambda_{j}^{2} ;|\Omega|^{2} \equiv \Omega^{T} \Omega=\sum_{j=1}^{m} \Omega_{j}^{2}$. The equation (3.1) is valid only under the condition

$$
\left(\lambda^{T} \Omega\right)^{2} \geq\left(\lambda^{T} \lambda\right) \cdot\left(\Omega^{T} \Omega\right)
$$

which can be satisfied only with equality sign, i. e. $\left(\lambda^{T} \Omega\right)^{2}=\left(\lambda^{T} \lambda\right) \cdot\left(\Omega^{T} \Omega\right)$ or

$$
J(b, s, \beta) \equiv \frac{\left(\lambda^{T} \Omega\right)^{2}}{\lambda^{T} \lambda \cdot \Omega^{T} \Omega}=1 .
$$

Because of modelling and measurements error, the value $J(b, s, \beta)$ remains always less than unity. Thus, a problem here is to maximise the function $J(b, s, \beta)$ with respect to boundary and damage parameters, i. e.

$$
\begin{aligned}
& E_{0}(b, s, \beta)=1-J(b, s, \beta) \equiv 1-\frac{\left(\lambda^{T} \Omega\right)^{2}}{\lambda^{T} \lambda \cdot \Omega^{T} \Omega} \Rightarrow \min \\
& F\left(\lambda_{j}, s, \beta, b\right)=0, \quad j=1, \ldots, m .
\end{aligned}
$$


Suppose that solution of the optimization problem (3.3) is $\hat{b}, \hat{s}, \hat{\beta}$ then $\hat{b}$ is accepted as corrected boundary parameter vector and corrected model parameter $\hat{a}$ can be found as

$$
\hat{a}=\left(\hat{\lambda}^{T} \Omega\right) / \Omega^{T} \Omega
$$

where $\hat{\lambda}=\lambda(\hat{b}, \hat{s}, \hat{\beta})$. Thus, the model updating has been completed and the obtained parameters will be used for correcting the measurement data. The model updated and the damage parameters estimated from the measured natural frequencies enables making correction of the measurement data $\Omega=\left(\Omega_{1}, \ldots, \Omega_{m}\right)^{T}=\left(\sqrt{\omega_{1}^{*}}, \ldots, \sqrt{\omega_{m}^{*}}\right)^{T}$ by calculating the frequency parameters

$$
\lambda_{j}^{*}=\hat{a} \Omega_{j}=\hat{a} \sqrt{\omega_{j}^{*}}, j=1, \ldots, m .
$$

The obtained vector $\lambda^{*}=\left(\lambda_{1}^{*}, \ldots, \lambda_{m}^{*}\right)^{T}$ representing the corrected measurement data certainly satisfies the conditions (3.1-3.2). If the natural frequencies of undamaged beam were measured, the optimisation problem (3.3) would be much more simplified by seeking only boundary parameters $\hat{b}$ and constrain equations in (3.3) now becomes $F\left(\lambda_{j}, s, \beta, b\right)=$ $F_{0}\left(\lambda_{j}, b\right)=0$.

\section{DAMAGE DETECTION}

The model updating and measurement correction described previously result in corrected model parameters such as $\hat{a}, \hat{b}$ and measurement data $\lambda^{*}=\left(\lambda_{1}^{*}, \ldots, \lambda_{m}^{*}\right)^{T}=\hat{a} \Omega$, which now are used for identification of damage parameter s, $\beta$. Firstly, the frequency equation (2.6) with given $\lambda=\left(\lambda_{1}, \ldots, \lambda_{m}\right)^{T}$ can be rewritten as

$$
\beta=d_{0 j} / d_{1 j}(s), j=1, \ldots, m,
$$

where $d_{0 j}=-F_{0}\left(\lambda_{j}, \hat{b}\right) ; d_{1 j}(s)=F_{1}\left(\lambda_{j}, s, \hat{b}\right)$ are components of vectors $d_{0}$ and $d_{1}$ respectively. The equations (4.1), as shown above, is equivalent to the equation

$$
d_{0}^{T} d_{0}-2 \beta d_{0}^{T} d_{1}+\beta^{2} d_{1}^{T} d_{1}=0
$$

that is meaningful with respect to the damage parameter $\beta$ only under the condition

$$
g(\lambda, s) \equiv\left(d_{0}^{T} d_{1}\right)^{2} /\left(d_{0}^{T} d_{0}\right) \cdot\left(d_{1}^{T} d_{1}\right)=1 .
$$

The latter equation with the corrected measurement data $\lambda^{*}=\left(\lambda_{1}^{*}, \ldots, \lambda_{m}^{*}\right)^{T}$ should be an exact equation for detecting damage sit $s$. Nevertheless, due to the measurement error, the function $g(\lambda, s)$ always remains less than 1 and the actual damage sit could only minimize the called here damage sit function $J_{1}(\lambda, s)=1-g(\lambda, s)$, so that the damage location leads to solving the problem

$$
\left\{s: \min J_{1}\left(\lambda^{*}, s\right),-0.5 \leq s \leq 0.5\right\} .
$$


Global solution of the problem (4.3) is ever-existing, say $\bar{s}$ with the global minimum $J_{1}^{*}=J_{1}\left(\lambda^{*}, \bar{s}\right)$. Furthermore, in order to detect damage magnitude $\beta$ with reduced further measurement error it has to solve the problem

$$
\begin{aligned}
& J_{2}(\lambda)=\sum_{j=1}^{m}\left(1-\lambda_{j} / \lambda_{j}^{*}\right)^{2} \Rightarrow \min , \\
& G(\lambda)=J_{1}(\lambda, \bar{s})-J_{1}^{*}=0 \\
& 0 \leq \lambda_{1} \leq \lambda_{2}^{*}, \lambda_{j-1}^{*} \leq \lambda_{j} \leq \lambda_{j+1}^{*}, j=2, \ldots, m-1 .
\end{aligned}
$$

If solution of the problem (4.4) has been found as $\bar{\lambda}_{1}, \ldots, \bar{\lambda}_{m-1}$, the magnitude of damage could be calculated as

$$
\bar{\beta}=-\frac{\sum_{j=1}^{m} F_{0}\left(\bar{\lambda}_{j}, \hat{b}\right) F_{1}\left(\bar{\lambda}_{j}, \bar{s}, \hat{b}\right)}{\sum_{j=1}^{m} F_{1}^{2}\left(\bar{\lambda}_{j}, \bar{s}, \hat{b}\right)} .
$$

The problem of damage detection for beam is thus solved.

\section{NUMERICAL CASE STUDY}

For illustration, the aluminium cantilever beam studied in [16] is taken under consideration herein based on the developed above theory. Initial model parameters and measurement data of the beam are as follow: the length $L=495.3 \mathrm{~mm}$, wide $W=25.4$ $\mathrm{mm}$, thick $H=6.35 \mathrm{~mm}$, Young's.modulus $E=7.1 \cdot 10^{7} \mathrm{kPa}$, mass density $\rho=2210 \mathrm{~kg} /$ $\mathrm{m}^{3}$. For the experimental prototype, the natural frequencies of intact as well as damaged beam have been measured, so that in both the cases the measured data can be determined as

$$
\begin{aligned}
& \Omega_{1}^{0}=11.07749 ; \Omega_{2}^{0}=27.6923 ; \Omega_{3}^{0}=46.16964 ; \Omega_{4}^{0}=64.48083 ; \Omega_{5}^{0}=82.57509 ; \Omega_{6}^{0}=100.0955 \\
& \Omega_{1}=10.92615 ; \Omega_{2}=26.9797 ; \Omega_{3}=45.69772 ; \Omega_{4}=63.75469 ; \Omega_{5}=80.7376 ; \Omega_{6}=99.99411
\end{aligned}
$$

Let's consider the cantilever with only two boundary parameters $b_{1}, b_{2}$ at the clamped end and, hence, only three measured frequencies of undamaged beam $\Omega_{0}=\left(\Omega_{j}^{0}, j=\right.$ $1,2,3)$ will be used for model updating. In this case, the shape functions $L_{j}$ have the form

$$
L_{1}(z)=K_{2}(z)-\lambda^{3} b_{1} K_{1}(z) ; \quad L_{2}(z)=K_{4}(z)+\lambda^{-1} b_{2} K_{3}(z) ; L_{3}(z)=K_{2}(z) ; L_{4}(z)=K_{4}(z) .
$$

Introducing the functions

$$
D_{11}=-\lambda^{4} \operatorname{det} A_{11}, D_{1}=-\lambda^{3} \operatorname{det} A_{10}, D_{2}=\lambda \operatorname{det} A_{01}, D_{0}=\operatorname{det} A_{00},
$$

with matrices $A_{11}, A_{10}, A_{01}, A_{00}$ given in Appendix, the frequency equation for undamaged beam now takes the form

$$
D_{11}(\lambda) b_{1} b_{2}+D_{1}(\lambda) b_{1}+D_{2}(\lambda) b_{2}+D_{0}(\lambda)=0 .
$$


Suppose that $\lambda_{1}, \lambda_{2}, \lambda_{3}$ are three solutions of the equation (5.1), i. e.

$$
D_{11}\left(\lambda_{j}\right) b_{1} b_{2}+D_{1}\left(\lambda_{j}\right) b_{1}+D_{2}\left(\lambda_{j}\right) b_{2}+D_{0}\left(\lambda_{j}\right)=0, j=1,2,3 .
$$

and that $\delta=\operatorname{det} \Delta, \delta_{1}=\operatorname{det} \Delta_{1}, \delta_{2}=\operatorname{det} \Delta_{2}, \delta_{3}=\operatorname{det} \Delta_{3}$ with the matrices $\Delta, \Delta_{1}, \Delta_{2}, \Delta_{3}$ given in Appendix, then, the equations (5.2) can be replaced by the conditions

$$
g\left(\lambda_{1}, \lambda_{2}, \lambda_{3}\right) \equiv \delta_{1} \delta_{2}-\delta \delta_{3}=0 \text { and } h\left(\lambda_{1}, \lambda_{2}, \lambda_{3}\right) \equiv \delta^{2}-\varepsilon \geq 0 .
$$

Thus, the model updating problem (3.3) leads to the standard problem of constrained programming

$$
\begin{aligned}
& J(\lambda)=1-\frac{\left(\lambda^{T} \Omega_{0}\right)^{2}}{\lambda^{T} \lambda} \times \Omega_{0}^{T} \Omega_{0} \Rightarrow \min , \\
& g(\lambda)=0 ; \quad h(\lambda) \geq 0 ; \quad \lambda_{1}-\varepsilon \geq 0 ; \quad \lambda_{j+1}-\lambda_{j}-\varepsilon \geq 0, j=1,2 .
\end{aligned}
$$

If $\hat{\lambda}=\left\{\hat{\lambda}_{1}, \hat{\lambda}_{2}, \hat{\lambda}_{3}\right\}$ is solution of the problem (5.4), the updated model parameters could be obtained as

$$
\hat{a}=\hat{\lambda}^{T} \Omega_{0} / \Omega_{0}^{T} \Omega_{0}, \hat{b}_{1}=\delta_{1}(\hat{\lambda}) / \delta(\hat{\lambda}), \hat{b}_{2}=\delta_{2}(\hat{\lambda}) / \delta(\hat{\lambda}) .
$$

The corrected measurement data for damage detection now can be computed by formula (3.5).

Using only the first three values from the measured data for the undamaged beam $\Omega_{0}$, the problem (5.3) has been solved by the MATLAB function FMIN$\mathrm{CON}$ and updated model parameters have been obtained as $\hat{a}=0.1677 ; \hat{b}_{1}=$ $1.265 \cdot 10^{-4} ; \hat{b}_{2}=0.069$ (the initial values of the model parameters $a=0.1537$, $\left.b_{1}=0.0, b_{2}=0.0\right)$. Therefore, instead of design bending stiffness $(E I)_{0}$ $=38.4799 \mathrm{Nm}^{2}$, its updated value now is $(E I)_{u}=27.1232 \mathrm{Nm}^{2}$ and boundary spring stiffness can be calculated as $S_{t}=1.7646 \mathrm{e} 06 \mathrm{~N} / \mathrm{m}, S_{r}=793.6397$ $\mathrm{Nm} / \mathrm{rad}$. The measurement calibration procedure is thus performed by calculating corrected measurement data accordingly to the equation (3.5) with the

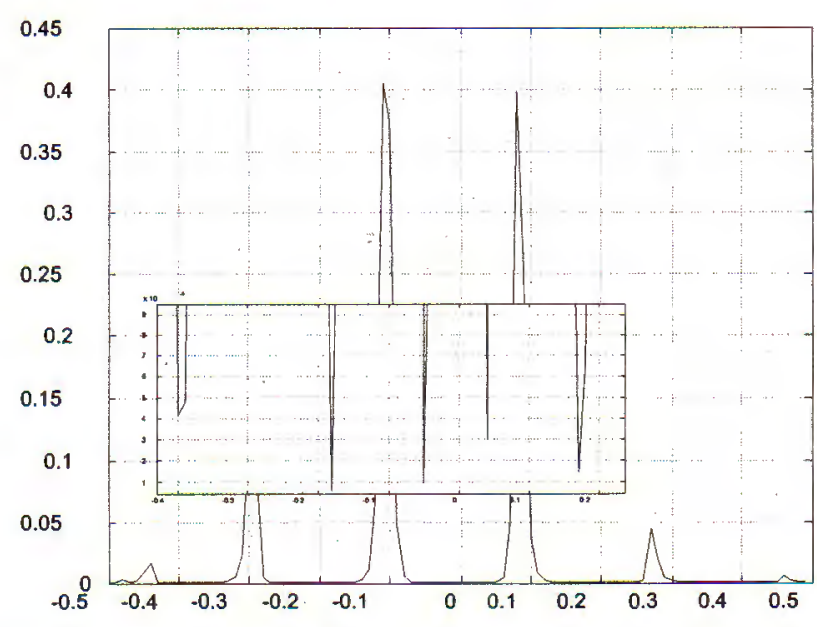

Fig. 1. The damage sit function with its local minimums updated model parameters. 
For detecting damage sit, numerical investigation of the function $J_{1}\left(\lambda^{*}, s\right)$ in different cases of correction of measurement data and model updating has been carried out. The results show that the function $J_{1}\left(\lambda^{*}, s\right)$ has unique zero at $\mathrm{s}=0.5$ (the free end) and many local minimums, from which the global one can be derived. However, the determining global minimum is not definitely because the local minimums cannot be clearly distinguished. In Table 1 there are listed five most clearly appeared local minimums of the function in the following cases: both the model updating and measurements correction (case 1); no model updating nor measurements correction (case 2); model updating only (case 3 ) and measurements correction only (case 4). Judging among the global minimums that bolded in Table 1 , it can be seen that only in the case 1, the global minimum $\bar{s}=-0.0487$ may be accepted as detected correspondingly damage position $x_{s}=22.3529$ $\mathrm{cm}$ compared with the actual damage position $20.63 \mathrm{~cm}$. Following the local minimums in comparison with the actual damage position, the best result in damage position detection $\left(\tilde{x}_{s}=21.8437 \mathrm{~cm}\right)$ has been obtained in the case of only model updating. It is reasonable to affirm that model updating is mandatory in damage detection otherwise it may lead to erroneous result of detection.

Table 1. Local minimums of the damage sit function in different cases of model updating and measured data correction

\begin{tabular}{|c|c|c|c|c|c|c|c|c|}
\hline \multirow{2}{*}{$\begin{array}{c}\text { Local } \\
\text { minimum }\end{array}$} & \multicolumn{2}{|c|}{ Case 1} & \multicolumn{2}{|c|}{ Case 2} & \multicolumn{2}{|c|}{ Case 3} & \multicolumn{2}{|c|}{ Case 4} \\
\hline & $\mathrm{s}$ & $J(s)$ & $\mathrm{s}$ & $J(s)$ & $\mathbf{s}$ & $J(s)$ & $\mathrm{s}$ & $\mathrm{J}(\mathrm{s})$ \\
\hline 1 & -0.3954 & $9.3830 \mathrm{E}-7$ & -0.4288 & $8.2546 \mathrm{E}-7$ & -0.4596 & $2.5226 \mathrm{E}-7$ & -0.4106 & $1.5504 \mathrm{E}-8$ \\
\hline 2 & -0.1797 & $5.5148 \mathrm{E}-7$ & -0.2373 & $1.5245 \mathrm{E}-5$ & -0.2799 & $1.9377 \mathrm{E}-5$ & -0.3155 & $5.8497 \mathrm{E}-7$ \\
\hline 3 & -0.0487 & $3.4088 \overline{E-9}$ & -0.1511 & $2.6775 \mathrm{E}-6$ & -0.0590 & $2.2981 \mathrm{E}-5$ & 0.1695 & $5.0097 \mathrm{E}-6$ \\
\hline 4 & 0.0385 & $4.2514 \mathrm{E}-7$ & -0.0210 & $1.4924 \mathrm{E}-5$ & $\overline{0.2680}$ & $1.2241 \mathrm{E}-5$ & $\overline{0.2774}$ & $1.5960 \mathrm{E}-5$ \\
\hline 5 & 0.1725 & $7.1811 \mathrm{E}-7$ & 0.2855 & $1.2110 \mathrm{E}-5$ & 0.3731 & $6.2019 \mathrm{E}-8$ & 0.3606 & $2.7288 \mathrm{E}-7$ \\
\hline & & & $a g$ & & in & -0.0835 & & \\
\hline
\end{tabular}

With damage position detected above in the case of model updating and measurement correction $(\bar{s}=-0.0487)$, the problem (4.4) has been solved and results in the damage magnitude equal to $\vec{\beta}=0.0126$. For the damage position detected $\tilde{x}_{s}=21.8437 \mathrm{~cm}$, solution of the problem (4.4) gives $\tilde{\beta}=0.0088$.

\section{CONCLUSION}

The main results presented in this paper are as follow:

1. The theoretical basics for analysis and identification of single damaged beam have been established that is usable for generalised boundary conditions included all the wellknown classical ones. The constructed analytical model of damaged beam is useful for damage detection due to that leads to the damage location separated from the damage magnitude detection, so that an equation for damage location is simply derived and solved in general case of boundary condition. At that time the damage detection problem is thus simplified and can be solved by a general procedure.

2. A general procedure for damage detection in beam including the model updating, the measured data calibration, the damage location and damage severity assessment is developed in general form. In the model updating stage, the model parameter such as geometrical, material constants as well as boundary conditions have been updated using measured data. The measured data are calibrated twice, first for damage location using 
the updated model parameters and boundary conditions and then for damage severity assessment using additionally the identified damage sit.

3. Illustrated example showed that behaviour of the damage sit function derived for damage location depending on the boundary condition may be very complicate, so that solution of the damage location problem requires specific investigation. From the numerical results it has been seen also that the model updating task is mandatory required whenever before solving the damage detection problem. The measured data are ever-erroneous, but they should be calibrated through the damage detection process.

This work was completed by financial support from the National Council in Natural Sciences of Vietnam

\section{APPENDIX}

\section{A.1. The boundary (shape) functions}

Firstly, for simplicity in writing, the Krylov's functions are used

$$
\begin{gathered}
K_{1}(z)=0.5(\cosh z+\cos z) ; K_{3}(z)=0.5(\cosh z-\cos z) \\
K_{2}(z)=0.5(\sinh z-\sin z) ; K_{4}(z)=0.5(\sinh z+\sin z) .
\end{gathered}
$$

Obviously, the functions have the properties

$$
\begin{aligned}
& K_{1}(0)=1, K_{2}(0)=K_{3}(0)=K_{4}(0)=0 \\
& K_{1}^{\prime}(z)=K_{2}(z), \quad K_{2}^{\prime}(z)=K_{3}(z), \quad K_{3}^{\prime}(z)=K_{4}(z), \quad K_{4}^{\prime}(z)=K_{1}(z) .
\end{aligned}
$$

On the other hand, the boundary functions are written in the form

$$
L_{j}(z)=L_{0 j}(z)+b_{j} L_{1 j}(z), j=1,2,3,4
$$

with the boundary parameters $b_{j}$ and functions $L_{0 j}, L_{1 j}$ defined below for different cases of generalized boundary condition (elastic supports).

Let's consider a beam with elastic supports at both ends. Each end is rested on two springs, one of which is translational and another is rotational. Stiffness of the springs is denoted respectively as $S_{t 0}, S_{r 0}$ for the left end and $S_{t 1}, S_{r 1}$ for the right one. The boundary conditions, in this case, have the following form

$E I w^{\prime \prime \prime}+S_{t 0} w=0$ and $E I w^{\prime \prime}-S_{r 0} w^{\prime}=0$, for the left boundary;

$E I w^{\prime \prime \prime}-S_{t 1} w=0$ and $E I w^{\prime \prime}+S_{r 1} w^{\prime}=0$, for the right boundary;

The flexural (bending) curve is taken as

$$
w(x)=A_{1} K_{1}(z)+A_{2} K_{2}(z)+A_{3} K_{3}(z)+A_{4} K_{4}(z) ; z=\lambda(x+0.5)
$$

for the left end and

$$
w(x)=A_{1} K_{1}(z)+A_{2} K_{2}(z)+A_{3} K_{3}(z)+A_{4} K_{4}(z) ; z=\lambda(x-0.5)
$$

for right one. Substituting the later expressions into the boundary conditions yields the required boundary functions. The boundary parameters $b_{j}$ are introduced so that their trivial value is corresponding to the classical boundary conditions. 
A.1.1 Generalised Hinges: In this case both the ends of beam are elastically supported by springs of stiffness denoted as $\mathrm{S}_{t 0}, \mathrm{~S}_{r 0}$ for the left end and $\mathrm{S}_{t 1}, \mathrm{~S}_{r 1}$ for the other one. The boundary parameters $\mathrm{b}$ are introduced here so that their zero values give the simply supported beam, i.e.

$$
b_{1}=\frac{E I}{S_{t 0} L^{3}} ; \quad b_{3}=\frac{E I}{S_{t 1} L^{3}} ; \quad b_{2}=\frac{S_{r 0} L}{E I} ; b_{4}=\frac{S_{r 1} L}{E I} .
$$

The boundary functions thus will be obtained in the form

$$
\begin{array}{ll}
L_{1}(z)=K_{2}(z)-\lambda^{3} b_{1} K_{1}(z) ; & L_{3}(z)=K_{2}(z)+\lambda^{3} b_{3} K_{1}(z) ; \\
L_{2}(z)=K_{4}(z)+\lambda^{-1} b_{2} K_{3}(z) ; & L_{4}(z)=K_{4}(z)-\lambda^{-1} b_{4} K_{3}(z) .
\end{array}
$$

Thus, in this case,

$$
\begin{aligned}
& L_{01}(z)=L_{03}(z)=K_{2}(z), L_{02}(z)=L_{04}(z)=K_{4}(z), \\
& L_{11}(z)=-L_{13}(z)=-\lambda^{3} K_{1}(z), L_{12}(z)=-L_{14}(z)=\lambda^{-1} K_{3}(z) .
\end{aligned}
$$

If all the parameters $b=0$, we get the functions for well known simply supported beam

$$
L_{1}(z)=L_{3}(z)=K_{2}(z) ; L_{2}(z)=L_{4}(z)=K_{4}(z) .
$$

In the later case, because of the symmetry of the boundary conditions, the functions could be simplified into $L_{1}(z)=L_{3}(z)=\sin z ; L_{2}(z)=L_{4}(z)=\sinh z$.

A.1.2 Generalised Clamps: For the reason mentioned above this case is differed from the generalised hinges case by introducing the boundary parameters

$$
b_{1}=\frac{E I}{S_{t 0} L^{3}} ; \quad b_{3}=\frac{E I}{S_{t 1} L^{3}} ; \quad b_{2}=\frac{E I}{S_{r 0} L} ; \quad b_{4}=\frac{E I}{S_{r 1} L} .
$$

The boundary functions will take the form

$$
\begin{aligned}
& L_{1}(z)=K_{2}(z)-\lambda^{3} b_{1} K_{1}(z), \quad L_{3}(z)=K_{2}(z)+\lambda^{3} b_{3} K_{1}(z), \\
& L_{2}(z)=K_{3}(z)+\lambda b_{2} K_{4}(z), \quad L_{4}(z)=K_{3}(z)-\lambda b_{4} K_{4}(z),
\end{aligned}
$$

that give finally the functions

$$
\begin{aligned}
& L_{01}(z)=L_{03}(z)=K_{2}(z), L_{02}(z)=L_{04}(z)=K_{3}(z) \\
& L_{11}(z)=-L_{13}(z)=-\lambda^{3} K_{1}(z), L_{12}(z)=-L_{14}(z)=\lambda K_{4}(z) .
\end{aligned}
$$

By the analogy, for the ideally clamped beam, corresponding to trivial value of the boundary parameters $b$, the boundary functions are simplified as

$$
L_{1}(z)=L_{3}(z)=K_{2}(z), L_{2}(z)=L_{4}(z)=K_{3}(z) .
$$

A.1.3 Generalised Cantilever: The ideal cantilever beam has the right end to be free and the left one to be clamped. In generalized cantilever beam case, for the elastically supported at both sides ends beam the following boundary non-dimensional parameters are introduced

$$
b_{1}=\frac{E I}{S_{t 0} L^{3}} ; b_{2}=\frac{E I}{S_{r 0} L} ; b_{3}=\frac{S_{t 1} L^{3}}{E I} ; b_{4}=\frac{S_{r 1} L}{E I} ;
$$


so that the boundary functions will have the form

$$
\begin{aligned}
& L_{1}(z)=K_{2}(z)-\lambda^{3} b_{1} K_{1}(z), \quad L_{3}(z)=K_{1}(z)+\lambda^{-3} b_{3} K_{2}(z) \\
& L_{2}(z)=K_{3}(z)+\lambda b_{2} K_{4}(z), \quad L_{4}(z)=K_{4}(z)-\lambda^{-1} b_{4} K_{3}(z) .
\end{aligned}
$$

Therefore,

$$
\begin{aligned}
& L_{01}(z)=K_{2}(z), L_{02}(z)=K_{3}(z), L_{03}(z)=K_{1}(z), L_{04}(z)=K_{4}(z), \\
& L_{11}(z)=-\lambda^{3} K_{1}(z), L_{12}(z)=\lambda K_{4}(z), L_{13}(z)=\lambda^{-3} K_{2}(z), L_{14}(z)=-\lambda^{-1} K_{3}(z) .
\end{aligned}
$$

A.2. The matrices $A_{11}, A_{10}, A_{01}, A_{00}, \Delta, \Delta_{1}, \Delta_{2}, \Delta_{3}$

$$
\begin{aligned}
& \xi=\lambda(s+0.5) ; \zeta=\lambda(s-0.5) \\
& A_{11}=\left[\begin{array}{cccc}
K_{1}(\xi) & K_{4}(\xi) & K_{1}(\zeta) & K_{4}(\zeta) \\
K_{2}(\xi) & K_{1}(\xi) & K_{2}(\zeta) & K_{1}(\zeta) \\
K_{3}(\xi) & K_{2}(\xi) & K_{3}(\zeta) & K_{2}(\zeta) \\
K_{4}(\xi) & K_{3}(\xi) & K_{4}(\zeta) & K_{3}(\zeta)
\end{array}\right], A_{10}=\left[\begin{array}{cccc}
K_{1}(\xi) & K_{3}(\xi) & K_{1}(\zeta) & K_{4}(\zeta) \\
K_{2}(\xi) & K_{4}(\xi) & K_{2}(\zeta) & K_{1}(\zeta) \\
K_{3}(\xi) & K_{1}(\xi) & K_{3}(\zeta) & K_{2}(\zeta) \\
K_{4}(\xi) & K_{2}(\xi) & K_{4}(\zeta) & K_{3}(\zeta)
\end{array}\right], \\
& A_{01}=\left[\begin{array}{llll}
K_{2}(\xi) & K_{4}(\xi) & K_{1}(\zeta) & K_{4}(\zeta) \\
K_{3}(\xi) & K_{1}(\xi) & K_{2}(\zeta) & K_{1}(\zeta) \\
K_{4}(\xi) & K_{2}(\xi) & K_{3}(\zeta) & K_{2}(\zeta) \\
K_{1}(\xi) & K_{3}(\xi) & K_{4}(\zeta) & K_{3}(\zeta)
\end{array}\right] ; A_{00}=\left[\begin{array}{cccc}
K_{2}(\xi) & K_{3}(\xi) & K_{1}(\zeta) & K_{4}(\zeta) \\
K_{3}(\xi) & K_{4}(\xi) & K_{2}(\zeta) & K_{1}(\zeta) \\
K_{4}(\xi) & K_{1}(\xi) & K_{3}(\zeta) & K_{2}(\zeta) \\
K_{1}(\xi) & K_{2}(\xi) & K_{4}(\zeta) & K_{3}(\zeta)
\end{array}\right], \\
& \Delta=\left[\begin{array}{lll}
D_{1}\left(\lambda_{1}\right) & D_{2}\left(\lambda_{1}\right) & D_{11}\left(\lambda_{1}\right) \\
D_{1}\left(\lambda_{2}\right) & D_{2}\left(\lambda_{2}\right) & D_{11}\left(\lambda_{2}\right) \\
D_{1}\left(\lambda_{3}\right) & D_{2}\left(\lambda_{3}\right) & D_{11}\left(\lambda_{3}\right)
\end{array}\right] ; \Delta_{1}=\left[\begin{array}{ccc}
-D_{0}\left(\lambda_{1}\right) & D_{2}\left(\lambda_{1}\right) & D_{11}\left(\lambda_{1}\right) \\
-D_{0}\left(\lambda_{2}\right) & D_{2}\left(\lambda_{2}\right) & D_{11}\left(\lambda_{2}\right) \\
-D_{0}\left(\lambda_{3}\right) & D_{2}\left(\lambda_{3}\right) & D_{11}\left(\lambda_{3}\right)
\end{array}\right] ; \\
& \Delta_{2}=\left[\begin{array}{lll}
D_{1}\left(\lambda_{1}\right) & -D_{0}\left(\lambda_{1}\right) & D_{11}\left(\lambda_{1}\right) \\
D_{1}\left(\lambda_{2}\right) & -D_{0}\left(\lambda_{2}\right) & D_{11}\left(\lambda_{2}\right) \\
D_{1}\left(\lambda_{3}\right) & -D_{0}\left(\lambda_{3}\right) & D_{11}\left(\lambda_{3}\right)
\end{array}\right] ; \Delta_{3}=\left[\begin{array}{lll}
D_{1}\left(\lambda_{1}\right) & D_{2}\left(\lambda_{1}\right) & -D_{0}\left(\lambda_{1}\right) \\
D_{1}\left(\lambda_{2}\right) & D_{2}\left(\lambda_{2}\right) & -D_{0}\left(\lambda_{2}\right) \\
D_{1}\left(\lambda_{3}\right) & D_{2}\left(\lambda_{3}\right) & -D_{0}\left(\lambda_{3}\right)
\end{array}\right] ;
\end{aligned}
$$

\section{REFERENCES}

1. A. Messina, E. J. Williams and T. Contursi, Structural damage detection by sensitivity and statistical-based method, Journal of Sound and Vibration 216 (5) (1998) 791-808.

2. R. D. Adams, P. Cawley, C. J. Pie and B. J. A. Stone, A vibration technique for nondestructively assessing the integrity of structures, Journal of Mechanical Engineering Science 20 (1978) 93-100.

3. T. G. Chondros, A. D. Dimarogonas and J. Yao, A continuous damaged beam vibration theory, Journal of Sound and Vibration 215 (1) (1998) 17-34.

4. P. F. Rizos, N. Aspragathos and A. D. Dimaroganas, Identification of crack location and magnitude in a cantilever beam from the vibration modes, Journal of Sound and Vibration 138 (3) (1990) 381-388.

5. Y. Narkis, Identification of crack location in vibrating simply supported beams, Journal of Sound and Vibration 172 (4) (1994) 549-558. 
6. M. Boltezar, B. Strancar and A. Kuhelj, Identification of transverse crack location in flexural vibration of free-free beams, Journal of Sound and Vibration 211 (5) (1998) 729-734.

7. S. Masoud, M. A. Jarran and M. Al-Maamory, Effect of crack depth on the natural frequency of a pre-stressed fixed-fixed beam, Journal of Sound and Vibration 214 (2) (1998) 201-212.

8. B. P. Nandwana and S. K. Maiti, Detection of the location and size of a crack in stepped cantilever beams based on measurements of natural frequencies, Journal of Sound and Vibration 203 (3) (1997) 435-446.

9. T. C. Tsai and Y. Z. Wang, Vibration analysis and diagnosis of a damaged shaft, Journal of Sound and Vibration 192 (3) (1996) 607-620.

10. H. P. Lin, Direct and inverse methods on free vibration analysis of simply supported beams with crack, Engineering Structures 26 (2004) 427-436.

11. R. Ruotolo and C. Surace, Damage assessment of multiple damaged beams: Numerical results and experimental validation, Journal of Sound and Vibration 206 (4) (1997) $567-588$.

12. N. T. Khiem and T. V. Lien, A simplified method for natural frequency analysis of multiple cracked beam, Journal of Sound and Vibration, 245 (4) (2001) 737-751.

13. R. Y. Liang, J. Hu and F. Choy, Theoretical study of crack-induced eigen-frequency changes on beam structures, Journal of Engineering Mechanics, ASCE, 118 (2) (1992), 384-396.

14. A. Morassi, Crack-induced changes in eigen-parameters of beam structures, Journal of Engineering Mechanics, ASCE, 119 (9) (1993) 1798-1803.

15. Nguyen Tien Khiem and Dao Nhu Mai, Natural frequency analysis of damaged beam, Journal of Mechanics, NCNST of Vietnam, 19 (2) (1997) 28-38..

16. S. Hassiotis and G. D. Jeong, Identification of stiffness reductions using natural frequencies, Journal of Engineering Mechanics, ASCE, 121 (10) (1995) 1106-1113. Discussion by S. Abrate, J. Spoerre and G. Schoeppner, Journal of Engineering Mechanics, ASCE, 123 (6) (1997) 647-650.

17. D. Armon, Y. Ben-Haim and S. Braun, Crack detection in beams by rank-ordering of eigen-frequency shifts, Mechanical Systems and Signal Processing 8 (1) (1994) 81-91.

18. H. T. Banks, D. J. Inman, D. J. Leo and Y. Wang, An experimentally validated damage detection theory in smart structures, Journal of Sound and Vibration 191 (5) (1996) 859-880.

Received April 28, 2006

\section{CHẦN DOÁN HU HÒNG, CƯA DẦM BẰNG TẦN SỐ RIÊNG: LÝ THUYẾT VÀ THUẠTT TOÁN}

Trong bài báo trình bày những phương trình cơ bàn để nghiên cứu dao động riêng của dầm đàn hồi có một hư hơng với các điều kiện biên tổng quát (gối mềm); một thuật toán để giải bài toán chẩn đoán hư hơng (vị trí và mức độ) bao gồm các công đoạn: điều chỉnh mô hình (điều kiện biên) và số liệu đo và xác định vị trí, mức độ hư hơng và một ví dụ số để minh họa cho lý thuyết và thuật toán. 